Pacific Journal of Mathematics

ELIMINATION OF MALITZ QUANTIFIERS IN STABLE 


\title{
ELIMINATION OF MALITZ QUANTIFIERS IN STABLE THEORIES
}

\begin{abstract}
ANDREAS RAPP
The main result of this paper is (a slightly stronger form of) the following theorem: let $T$ be a countable complete first-order theory which is stable. If, for some $\alpha \geq 1$, the Malitz quantifier $Q_{\alpha}^{2}$ is eliminable in $T$ then all Malitz quantifiers $Q_{\beta}^{m}(\beta \geq 0, m \geq 1)$ are eliminable in $T$. This complements results of Baldwin-Kueker [1] and Rothmaler-Tuschik [3].
\end{abstract}

1. Introduction. In this paper we consider various logics extending first-order logic that are obtained by adding quantifiers asserting the existence of a large homogeneous set of $n$-tuples. For $\alpha \geq 0, m, n \geq 1$, $Q_{\alpha}^{m, n}$ is a quantifier binding $m \cdot n$ variables whose semantics is defined by

$$
\begin{aligned}
\mathfrak{U} \vDash & Q_{\alpha}^{m, n} \bar{x}_{1} \cdots \bar{x}_{m} \varphi\left(\bar{x}_{1}, \ldots, \bar{x}_{m}\right) \\
& \text { iff there is a set } X \subset A^{n} \text { of power } \geq \aleph_{\alpha} \\
& \text { which is homogeneous for } \varphi, \text { i.e., for all } n \text {-tuples } \\
& \bar{b}_{1}, \ldots, \bar{b}_{m} \in X: \mathfrak{A} \vDash \varphi\left[\bar{b}_{1}, \ldots, \bar{b}_{m}\right] .
\end{aligned}
$$

The quantifiers $Q_{\alpha}^{m, n}$ were introduced by Baldwin and Kueker in [1] and denoted by $Q_{\alpha}^{* m, n}$. For $n=1, Q_{\alpha}^{m, n}$ is the usual Malitz quantifier of order $m$ in the $\aleph_{\alpha}$-interpretation, and $Q_{\alpha}^{1,1}$ is just the cardinality quantifier $Q_{\alpha}$ ("there are $\aleph_{\alpha}$ many"). We shall also consider another family of quantifiers: for $\alpha \geq 0$ and $n \geq 1, E_{\alpha}^{n}$ is defined by

$$
\begin{aligned}
\mathfrak{U} \vDash & E_{\alpha}^{n} x_{1} \cdots x_{n} y_{1} \cdots y_{n} \varphi(\bar{x}, \bar{y}) \\
& \quad \text { iff } \varphi \text { is an equivalence relation on } A^{n} \text { of index } \geq \aleph_{\alpha} .
\end{aligned}
$$

The statement on the right side is expressible by a sentence of $L\left(Q_{\alpha}^{2, n}\right)$, but there is no $L\left(Q_{\alpha}^{1, n}\right)$-sentence equivalent to it. So, being strictly stronger than $Q_{\alpha}^{1, n}, E_{\alpha}^{n}$ may be viewed as a weak form of $Q_{\alpha}^{2, n}$.

Adjoining a quantifier $Q_{\alpha}^{m, n}$ to an elementary logic $L$ results in an increase of expressive power. There are, however, first-order theories that admit elimination of $Q_{\alpha}^{m, n}$ : for each $L$-formula $\varphi\left(\bar{x}_{1}, \ldots, \bar{x}_{m}, \bar{y}\right)$, where $l\left(\bar{x}_{1}\right)=\cdots=l\left(\bar{x}_{m}\right)=n$, there is another $L$-formula $\delta(\bar{y})$ such that, for all models $\mathfrak{A} \vDash T$ of power $\geq \boldsymbol{\aleph}_{\alpha}$ and all $\bar{a} \in A$,

$$
(\mathfrak{A}, \bar{a}) \vDash \delta \leftrightarrow Q_{\alpha}^{m, n} \bar{x}_{1} \cdots \bar{x}_{m} \varphi .
$$


Throughout this paper $T$ always denotes a countable complete first-order theory with infinite models. For simplicity we assume that the language of $T$ does not have function symbols.

If $Q_{\alpha}^{m, n}$ is eliminable in $T$, this has interesting consequences. Firstly, $T$ remains complete as an $L\left(Q_{\alpha}^{m, n}\right)$-theory. Secondly, if $T$ is decidable and the elimination of $Q_{\alpha}^{m, n}$ can be carried out effectively, then it is also decidable whether a given $L\left(Q_{\alpha}^{m, n}\right)$-sentence holds in some (all) model(s) of $T$. In many cases elimination procedures are known; for a survey see [2].

Aside from investigating particular examples, it is natural to look for purely first-order properties of $T$ which imply or characterize eliminability of certain quantifiers $Q_{\alpha}^{m, n}$. Another problem is to determine the relative strength of eliminability of various $Q_{\alpha}^{m, n}$. Regarding the $\boldsymbol{\aleph}_{0}$-interpretation Baldwin and Kueker [1] gave a solution to both problems if stability of $T$ is assumed.

THEOREM 1.1. (a) If $T$ does not have the finite cover property (f.c.p.) then all quantifiers $Q_{0}^{m, n}$ for $m, n \geq 1$ are eliminable in $T$.

(b) If $T$ is stable and $E_{0}^{1}$ is eliminable in $T$ then $T$ does not have the f.c.p.

Thus Theorem 1.1. shows that in the stable case the following are equivalent:

(i) $T$ does not have the f.c.p.,

(ii) all quantifiers $Q_{0}^{m, n}$ and $E_{0}^{n}$ are eliminable in $T$;

(iii) any single quantifier $Q_{0}^{m, n}$ or $E_{0}^{n}(m \geq 2)$ is eliminable in $T$.

Regarding $\boldsymbol{\aleph}_{\alpha}$-interpretations for $\alpha \geq 1$ one has to look for a first-order property different from "not f.c.p." in order to characterize eliminability of $Q_{\alpha}^{m, n}$ or $E_{\alpha}^{n}$. For example, put $T:=\operatorname{Th}(A, R)$, where $R$ is an equivalence relation on $A$ with infinitely many equivalence classes and each class infinite. $T$ is $\omega$-categorical and $\omega$-stable. Hence, by Theorem 7 of [1], it admits elimination of all $Q_{0}^{m, n}$. Thus, by Theorem 1.1, $T$ does not have the f.c.p. However, none of $E_{\alpha}^{n}$ or $Q_{\alpha}^{m, n}$ for $\alpha \geq 1$ is eliminable in $T$.

In [3] Rothmaler and Tuschik introduced the notion of a regular theory and proved

THEOREM 1.2. If $T$ is regular then all quantifiers $Q_{\alpha}^{m, n}(\alpha \geq 0, m \geq 1)$ are eliminable in $T$.

It is also mentioned in their paper ("added in proof") that, for stable $T$, the converse of Theorem 1.2 is true. This suggests regularity as a 
substitute for "not f.c.p." when looking at all Malitz quantifiers $Q_{\alpha}^{m, n}$. Yet, when this result is compared to Theorem 1.1, two questions remain open:

(1) Does regularity of $T$ imply eliminability of $Q_{\alpha}^{m, n}$ for $n>1$ ?

(2) Is there a single quantifier whose eliminability implies that of all $Q_{\alpha}^{m, n}$ ?

It will turn out (see Corollary 3.4 ) that, in the stable case, question 1 may be answered positively. When using a slightly more general concept than regularity, which we call strong regularity, the stability assumption can be dropped (cf. Definition 2.3 and Theorem 2.4):

THEOREM 1.3. If $T$ is strongly regular then all quantifiers $Q_{\alpha}^{m, n}(\alpha \geq 0$; $m, n \geq 1$ ) are eliminable in $T$.

This theorem is also due to Rothmaler and Tuschik (compare the appendix of [3]).

With regard to question 2 the main result of the present paper shows that the situation closely parallels that of $1.1(\mathrm{~b})$.

MAIN TheOREM (see 3.2). Let $T$ be stable and suppose some quantifier $Q_{\alpha}^{m, n}$ or $E_{\alpha}^{n}($ where $\alpha \geq 1$ and $m \geq 2$ ) is eliminable in $T$. Then $T$ is strongly regular.

Hence, for stable $T$, the following are equivalent:

(i) $T$ is strongly regular;

(ii) all quantifiers $Q_{\alpha}^{m, n}$ are eliminable in $T$,

(iii) any single quantifier $E_{\alpha}^{n}$ or $Q_{\alpha}^{m, n}(m \geq 2, \alpha \geq 1)$ is eliminable in $T$.

The main theorem partially answers problems 2 and 3 of [2]. It will be proved in $\S 3$. Section 2 contains definitions and known results. Moreover it is shown that the connections among eliminability of $E_{\alpha}^{n}$ for various $\alpha$ are exactly the same as for the cardinality quantifiers $Q_{\alpha}$. We thus obtain a generalization of some of Tuschik's results in [5].

2. We shall sometimes abbreviate the statement that a quantifier $Q$ is eliminable in a theory $T$ by $E L_{T}(Q)$. If, for any generalized quantifier $Q$ and $Q^{\prime}, L(Q)$ is included in $L\left(Q^{\prime}\right)$ then $E L_{T}\left(Q^{\prime}\right)$ implies $E L_{T}(Q)$. We shall collect some facts that are based on this simple observation.

Proposition 2.1.

(i) If $m \leq m^{\prime}$ and $n \leq n^{\prime}$ then $E L_{T}\left(Q_{\alpha}^{m^{\prime}, n^{\prime}}\right)$ implies $E L_{T}\left(Q_{\alpha}^{m, n}\right)$,

(ii) If $n \leq n^{\prime}$ then $E L_{T}\left(E_{\alpha}^{n^{\prime}}\right)$ implies $E L_{T}\left(E_{\alpha}^{n}\right)$, 
(iii) If $E L_{T}\left(E_{\alpha}^{n}\right)$ then $E L_{T}\left(Q_{\alpha}^{1, n}\right)$,

(iv) $E L_{T}\left(Q_{\alpha}^{1, n}\right)$ if and only if $E L_{T}\left(Q_{\alpha}^{1,1}\right)$.

Proof. Observe, for instance:

for (i):

$$
\begin{aligned}
& \vDash Q_{\alpha}^{m, n} x_{11} \cdots x_{m n} \varphi\left(\bar{x}_{1}, \ldots, \bar{x}_{m}\right) \leftrightarrow \exists u Q_{\alpha}^{m, n+1} \bar{x}_{1} y_{1} \cdots \bar{x}_{m} y_{m} \\
& \quad\left(\varphi\left(\bar{x}_{1}, \ldots, \bar{x}_{m}\right) \wedge \bigwedge_{1 \leq i \leq m} u=y_{i}\right) ;
\end{aligned}
$$

for (iii):

$\vDash Q_{\alpha}^{1, n} \bar{x} \varphi(\bar{x}) \leftrightarrow E_{\alpha}^{n} \bar{x} \bar{y}[(\varphi(\bar{x}) \wedge \varphi(\bar{y}) \wedge “ \bar{x}=\bar{y} ") \vee(\neg \varphi(\bar{x}) \wedge \neg \varphi(\bar{y}))] ;$

for (iv):

$$
\begin{aligned}
& \vDash Q_{\alpha}^{1,1} x \varphi(x) \leftrightarrow Q_{\alpha}^{1, n} \bar{x} \bigwedge_{1 \leq i \leq n} \varphi\left(x_{l}\right) ; \\
& \vDash Q_{\alpha}^{1, n} \bar{x} \varphi(\bar{x}) \leftrightarrow Q_{\alpha}^{1,1} y(“ y \in \operatorname{field}(\varphi) ”) .
\end{aligned}
$$

If $\varphi\left(x_{1}, \ldots, x_{n}, y_{1}, \ldots, y_{n}\right)$ is a first-order formula then eq $(\varphi)$ stands for the first-order sentence expressing that $\varphi$ is an equivalence relation on $n$-tuples. The number of equivalence classes is denoted by ind $(\varphi)$.

For $m, n, k<\omega$ and a formula $\delta\left(\bar{x}_{1}, \ldots, \bar{x}_{m}\right)$, where $l\left(\bar{x}_{1}\right)=\cdots=$ $l\left(\bar{x}_{m}\right)=n, H_{k}^{m, n} \bar{x}_{1} \cdots \bar{x}_{m} \delta$ stands for the first-order sentence asserting the existence of a homogeneous set of $n$-tuples for $\delta$ that contains at least $k$ such $n$-tuples.

The following lemma serves as a basic tool for eliminability investigations. In its general form it is due to Tuschik, for a proof see [2]. We state the lemma for the quantifiers $Q_{\alpha}^{m, n}$ and $E_{\alpha}^{n}$ : it shows that if they are eliminable in some theory $T$, then this can be done in a very simple way.

LEMMA 2.2 (Definability lemma).

(a) $E L_{T}\left(Q_{\alpha}^{m, n}\right)$ iff for each first-order formula $\delta\left(\bar{x}_{1}, \ldots, \bar{x}_{m}, \bar{z}\right)$ there is a number $k<\omega$ such that

$$
T \cup\left\{Q_{\alpha} y(y=y)\right\} \vDash \forall \bar{z}\left(H_{k}^{m, n} \bar{x}_{1} \cdots \bar{x}_{m} \delta \rightarrow Q_{\alpha}^{m, n} \bar{x}_{1} \cdots \bar{x}_{m} \delta\right) .
$$

(b) $E L_{T}\left(E_{\alpha}^{n}\right)$ iff for each first-order formula $\varphi(\bar{x}, \bar{y})$, where $l(\bar{x})=$ $l(\bar{y})=n$, there is $k<\omega$ such that

$$
T \cup\left\{Q_{\alpha} y(y=y)\right\} \vDash \forall \bar{z}\left(\mathrm{eq}(\varphi) \wedge(\varphi) \geq k \rightarrow E_{\alpha}^{n} \bar{x} \bar{y} \varphi\right) .
$$

DEFINITION 2.3. Let $L$ be a first-order language.

(i) A structure $\mathfrak{U}$ for $L$ is called $(m, n)$-singular if there are (a) an $L$-formula $\varphi\left(\bar{x}_{1}, \ldots, \bar{x}_{m}, \bar{y}\right)$ with $l\left(\bar{x}_{i}\right)=n$ for $1 \leq i \leq m$, 
(b) $\bar{a} \in A$ and $C \subset A^{n}$, where $\boldsymbol{\aleph}_{0} \leq|C|<|A|$,

such that $C$ is maximally homogeneous for $\varphi$ in $(\mathfrak{A}, \bar{a})$.

(ii) $T$ is $(m, n)$-regular if it has no $(m, n)$-singular models.

(iii) $T$ is regular if it is $(m, 1)$-regular for all $m$;

$T$ is strongly regular if it is $(m, n)$-regular for all $m, n \geq 1$.

(iv) $(\mathfrak{A}, \mathfrak{B})$ is a generalized Vaughtian pair of index $(m, n)$ if $\mathfrak{A} \prec \mathfrak{B}$, $A \neq B$, and for some formula $\varphi\left(\bar{x}_{1}, \ldots, \bar{x}_{m}, \bar{y}\right)$ there are $\bar{a} \in A$ and $C \subset A^{n}$ such that $C$ is an infinite maximally homogeneous set for $\varphi$ in $(\mathfrak{B}, \bar{a})$.

A straightforward application of the arguments in [3] to sequences rather than elements yields

THEOREM 2.4 ( Rothmaler, Tuschik).

(i) Let $\varphi\left(\bar{x}_{1}, \ldots, \bar{x}_{m}, \bar{y}\right)$ be an L-formula $T$ has a generalized Vaughtian pair of index $(m, n)$ for $\varphi$ iff $T$ has an $(m, n)$-singular model of power $\aleph_{1}$ for $\varphi$ iff $T$ has any $(m, n)$-singular model for $\varphi$.

(ii) If $T$ is $(m, n)$-regular then $Q_{\alpha}^{m, n}$ is eliminable in $T$ for all $\alpha \geq 0$.

Clearly, (ii) entails Theorems 1.2 and 1.3. In general, a formula $\varphi\left(\bar{x}_{1}, \ldots, \bar{x}_{m}\right)$ where $m>1$ may have maximal homogeneous sets of different cardinality in a model $\mathfrak{U}$. However, if $m=1$ then there is just one, namely $\varphi^{\mathfrak{A}}$. Similarly, if $\delta(\bar{x}, \bar{y})$ is an equivalence relation on $A^{n}$, then a maximally homogeneous set for $\neg \delta$ is just a set of representatives, and, clearly, all such sets have the same cardinality.

DEFINITION 2.5. $T$ is n-regular for equivalence relations if whenever $\mathfrak{U} \vDash T, \bar{a} \in A$ and $(\mathfrak{U}, \bar{a})=\mathrm{eq}\left(\delta\left(\bar{x}_{1}, \bar{x}_{2}, \bar{z}\right)\right)$, where $l\left(\bar{x}_{1}\right)=l\left(\bar{x}_{2}\right)=n$, then either ind $\left(\delta^{\mathfrak{A}}\right)<\omega$ or $\operatorname{ind}\left(\delta^{\mathfrak{A}}\right)=|A|$.

COROLlaRY 2.6. $T$ is n-regular for equivalence relations if and only if $E_{\alpha}^{n}$ is eliminable in $T$ for all $\alpha \geq 0$.

Proof. The direction from left to right is shown exactly like Theorem 2.4(ii). For the other one use the remark preceding Definition 2.5.

The remainder of this section is devoted to generalizing Tuschik's result on the relative strength of eliminability of $Q_{\alpha}$ to the quantifiers $E_{\alpha}^{1}$. Recall that, by Lemma 2.1(iv), $E L_{T}\left(Q_{\alpha}^{1, n}\right)$ if and only if $E L_{T}\left(Q_{\alpha}\right)$; so the situation does not change if $Q_{\alpha}$ is replaced by $Q_{\alpha}^{1, n}$ for some $n>1$. The 
same is true for $E_{\alpha}^{1}$ and $E_{\alpha}^{n}$ although this is not so obvious at first sight (see Proposition 3.1).

Proposition 2.7.

(i) $E L_{T}\left(E_{\alpha}^{1}\right)$ for some $\alpha>0$ implies $E L_{T}\left(E_{0}^{1}\right)$,

(ii) $E L_{T}\left(E_{1}^{1}\right)$ implies $E L_{T}\left(E_{\alpha}^{1}\right)$ for all $\alpha$.

Proof. (i) By the definability Lemma 2.2, for each formula $\varphi(\bar{x}, \bar{y}, \bar{z})$ there is a number $k$ such that, for all $\mathfrak{A} \vDash T$ of power $\geq \aleph_{\alpha}$ and all $\bar{a} \in A$, the following holds:

$$
\text { if } \operatorname{ind}\left(\varphi^{(\mathfrak{A}, \bar{a})}\right) \geq k \quad \text { then } \quad \operatorname{ind}\left(\varphi^{(\mathfrak{A}, \bar{a})}\right) \geq \boldsymbol{\aleph}_{\alpha} .
$$

Suppose there are $\mathfrak{B} \vDash T, \bar{b} \in B$ such that $(\mathfrak{B}, \bar{b}) \vDash \mathrm{eq}(\varphi)$ and $k \leq$ $\operatorname{ind}\left(\varphi^{(\mathscr{B}, \bar{b})}\right)<\boldsymbol{\aleph}_{0}$. By hypothesis $|B|<\boldsymbol{\aleph}_{\alpha}$. But $(\mathfrak{B}, \bar{b})$ has an elementary extension $(\mathfrak{S}, \bar{b})$ of power $\boldsymbol{\aleph}_{\alpha}$. Now $k \leq \operatorname{ind}\left(\varphi^{(\mathfrak{B}, \bar{b})}\right)=\operatorname{ind}\left(\varphi^{(\mathfrak{C}, \bar{b})}\right)<\boldsymbol{\aleph}_{0}$ $<\boldsymbol{\aleph}_{\alpha}$, a contradiction.

(ii) By (i), $E L_{T}\left(E_{0}^{1}\right)$. Assuming that $E_{\alpha}^{1}$ is not eliminable in $T$ for some $\alpha>1$, we can conclude that there are a model $\mathfrak{A} \vDash T$, such that $|A| \geq \aleph_{\alpha}$, and a singular equivalence relation on $A$ defined by some formula $\varphi(x, y, \bar{z})$ in an expansion $(\mathfrak{U}, \bar{a})$. Put $T^{\prime}:=\operatorname{Th}\left(\mathfrak{A}, \bar{a}, P^{\mathfrak{A}}\right)$, where the new predicate symbol $P$ is interpreted in $\mathfrak{A}$ by a set of representatives for $\varphi$. By Vaught's two-cardinal theorem, $T^{\prime}$ has a singular model of power $\aleph_{1}$, whence $E_{1}^{1}$ is not eliminable in $T$.

Thus $E L_{T}\left(E_{1}^{1}\right)$ is the strongest notion, $E L_{T}\left(E_{0}^{1}\right)$ the weakest, and $E L_{T}\left(E_{\alpha}^{1}\right)$ for $\alpha>1$ is somewhere in between. Under additional assumptions we can say more:

Corollary $2.8(G C H)$. If $\aleph_{\alpha}$ is regular and $E L_{T}\left(E_{\alpha+1}^{1}\right)$ then $E L_{T}\left(E_{1}^{1}\right)$.

Proof. Use Chang's two-cardinal theorem in the proof of 2.7(ii).

THEOREM 2.9. Let $T$ be stable. If $E L_{T}\left(E_{\alpha}^{1}\right)$ for some $\alpha>1$ then $E L_{T}\left(E_{1}^{1}\right)$.

Proof. Again, the hypothesis together with the negation of the claim imply that there is $\mathfrak{A} \vDash T$, and some formula $\varphi(x, y, \bar{z})$ defines a singular equivalence relation in some expansion $(\mathfrak{A}, \bar{a})$. We may assume that $|A|=\aleph_{1}$.

In order to finish the proof by contradiction, we have to verify that there is a model $\mathfrak{B} \vDash T$ of power $\aleph_{\alpha}$ such that, for some $\bar{b} \in B, \varphi$ defines a singular equivalence relaton in $(\mathfrak{B}, \bar{b})$. We would like to apply Shelah's 
two-cardinal theorem [4, p. 287] to $T^{\prime}=\operatorname{Th}\left(\mathfrak{A}, \bar{a}, P^{\mathfrak{A}}\right)$ as above, but due to new dependencies caused by $P^{\mathfrak{A}}, T^{\prime}$ may not be stable any longer. However, Shelah's method of "imaginary elements" provides a means to overcome this difficulty.

First, we may assume w.l.o.g. that the singular equivalence relation in $\mathfrak{U}$ is defined (without parameters) by a two-place relation symbol $R$, where $R \in L$ and $L$ is the language of $\mathfrak{A}$. Now let $L^{+}:=L \cup\{P, E\}$ and $\mathfrak{A}^{+}$be the following model for $L^{+}$:

(a) as domain $A \cup X$, where $X \cap A=\varnothing$ and $X$ contains exactly one element for each equivalence class of $R^{\mathfrak{A}}$,

(b) $P^{\mathfrak{A}^{+}}=A$ and the relations of $L$ are restricted to $P^{\mathfrak{A}^{+}}$,

(c) $E^{\mathfrak{A}^{+}}=\left\{(a, x) \mid a \in A, x \in X, a\right.$ is in the $R^{\mathfrak{A}}$-class coded by $\left.x\right\}$. Put $T^{+}:=\operatorname{Th}\left(\mathfrak{A}^{+}\right)$. Some obvious facts about $T^{+}$are the following:

(1) each $\mathfrak{A} \vDash T$ has, up to isomorphism, a unique extension $\mathfrak{A}^{+} \vDash T^{+}$;

(2) if $\mathfrak{B} \vDash T^{+}$then $\mathfrak{B}^{-1} \vDash T$, where $\mathfrak{B}^{-1}=P^{\mathfrak{B}} \uparrow L$. Hence $\left(\mathfrak{B}^{-1}\right)^{+}=\mathfrak{B}$.

Moreover, we have

LEMMA 2.10. $T^{+}$is stable.

From this, Theorem 2.9 is proved as follows: apply Shelah's twocardinal theorem to $T^{+} .\left(\mathfrak{A}^{+},(\neg P)^{\mathfrak{A}^{+}}\right)$is a model of type $\left(\boldsymbol{\aleph}_{1}, \boldsymbol{\aleph}_{0}\right)$, so there exists $\mathfrak{B} \vDash T^{+}$, where $\mathfrak{B}$ is of type $\left(\boldsymbol{\aleph}_{\alpha}, \aleph_{0}\right)$. Now $\mathfrak{B}^{-1}$ is the required model of $T$.

Proof of Lemma 2.10 (sketch). The first step is to show that $T^{+}$is a conservative extension of $T$ : for each $L^{+}$-formula $\varphi\left(x_{1}, \ldots, x_{n}\right)$ there is an $L$-formula $\pi(\bar{x})$ such that

$$
\begin{aligned}
& T^{+} \vDash \forall \bar{x}\left(\bigwedge_{i \leq n} P x_{i} \rightarrow(\varphi \leftrightarrow \pi)\right), \\
& T^{+} \vDash \forall \bar{x}\left(\pi(\bar{x}) \rightarrow \bigwedge_{i \leq n} P x_{i}\right),
\end{aligned}
$$

for all $\mathfrak{B} \vDash T^{+}: \mathfrak{B} \vDash \pi[\bar{c}]$ iff $\quad \mathfrak{B}^{-1} \vDash \pi[\bar{c}]$.

$\pi$ is defined by induction on $L^{+}$-formulas. The idea is that quantification over elements in $(\neg P)$ can be replaced by quantification over the corresponding $R$-equivalence classes.

Now assume $T^{+}$is not stable in $\lambda$. Then, for some $\mathfrak{M} \vDash T^{+}$, there is a subset $N \subset M,|N| \leq \lambda$ and $\mathfrak{M}$ realizes $\lambda^{+} L^{+}$-types over $N$. W.1.o.g. $N \subset P^{M}$. By (*) one can conclude that $\mathfrak{M}^{-1}$ realizes $\lambda^{+} L$-types over $N$, whence $T$ is not stable in $\lambda$. 
3. In this section we prove the main theorem of this paper. We need another fact about the quantifiers $E_{\alpha}^{n}$.

Proposition 3.1. For all $n>1$ :

(i) $L\left(E_{0}^{n}\right)$ is included in $L\left(E_{0}^{1}\right)$;

(ii) for all $\alpha \geq 0, E L_{T}\left(E_{\alpha}^{1}\right)$ implies $E L_{T}\left(E_{\alpha}^{n}\right)$.

Proof. Let $\varphi\left(\bar{x}, \bar{x}_{2}\right)$ be a first-order formula with $l\left(\bar{x}_{1}\right)=l\left(\bar{x}_{2}\right)=n$. We define

$$
\pi\left(y_{1}, y_{2}\right):=\forall \bar{w}\left[\exists \bar{v}_{1} \varphi\left(y_{1} \frown \bar{v}_{1}, \bar{w}\right) \leftrightarrow \exists \bar{v}_{2} \varphi\left(y_{2} \frown \bar{v}_{2}, \bar{w}\right)\right] .
$$

Let $\mathfrak{A}$ be a model of $T \cup\{\operatorname{eq}(\varphi)\}$ and let $K\left(\varphi^{\mathfrak{A}}\right)$ be the set of all $\varphi^{\mathfrak{A}}$-equivalence classes. For $b \in A$ put

Then:

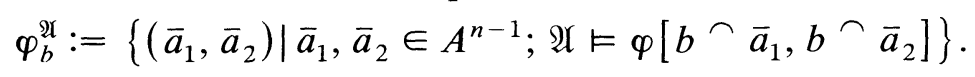

(1) $\varphi_{b}^{\mathfrak{A}}$ is an equivalence relation on $A^{n-1}$ and $K\left(\varphi_{b}^{\mathfrak{Q}}\right)$ consists essentially of all those $k \in K\left(\varphi^{\mathfrak{A}}\right)$ that contain some $n$-tuple $a_{1}, \ldots, a_{n}$ with $a_{1}=b$. Therefore, $\operatorname{ind}\left(\varphi_{b}^{\mathfrak{A}}\right) \leq \operatorname{ind}\left(\varphi^{\mathfrak{A}}\right)$.

(2) $K\left(\varphi^{\mathfrak{A}}\right)=\bigcup_{b \in A} K\left(\varphi_{b}^{\mathfrak{H}}\right)$.

(3) $\pi^{\mathfrak{A}}$ is an equivalence relation on $A$. For $b \in A$ the $\pi^{\mathfrak{A}}$-equivalence class of $b$ is completely determined by the set $K\left(\varphi_{b}^{\mathfrak{A}}\right)$. Hence, ind $\left(\pi^{\mathfrak{A}}\right) \leq$ $2^{\text {ind }\left(\varphi^{2}\right)}$.

Now we claim

$$
T \vDash E_{0}^{n} \bar{x}_{1} \bar{x}_{2} \varphi \leftrightarrow
$$

$$
\left[\operatorname{eq}(\varphi) \wedge\left(\exists z E_{1}^{n-1} \bar{v}_{1} \bar{v}_{2} \varphi\left(z \frown \bar{v}_{1}, z \frown \bar{v}_{2}\right) \vee E_{0}^{1} y_{1} y_{2} \pi\left(y_{1}, y_{2}\right)\right)\right] .
$$

Let $\mathfrak{A} \vDash E_{0}^{n} \bar{x}_{1} \bar{x}_{2} \varphi$. Then $\operatorname{ind}\left(\varphi^{\mathfrak{A}}\right)=\left|K\left(\varphi^{\mathfrak{A}}\right)\right| \geq \boldsymbol{\aleph}_{0}$. Now either some $K\left(\varphi_{b}^{\mathfrak{A}}\right)$ is infinite or otherwise (2) implies $K\left(\pi^{\mathfrak{A}}\right)$ to be infinite.

For the other direction, suppose $\varphi^{\mathscr{A}}$ is an equivalence relation of finite index. By (1), ind $\left(\varphi_{b}^{\mathscr{A}}\right)<\boldsymbol{\aleph}_{0}$ for each $b \in A$. By (3), ind $\left(\pi^{\mathfrak{A}}\right) \leq 2^{\operatorname{ind}\left(\varphi^{\mathscr{A}}\right)}<$ $\aleph_{0}$. So the right side of $(*)$ is false in $\mathfrak{A}$.

From (*) it follows by induction on $n$ that (i) of the proposition holds. Also, (ii) is clear for $\alpha=0$. Now assume that, for some $\alpha \geq 1, E L_{T}\left(E_{\alpha}^{1}\right)$ holds and $E L_{T}\left(E_{\alpha}^{n}\right)$ does not, where $n>1$ is minimal with this property. By 2.7(i) and 3.1(i) above, $E L_{T}\left(E_{0}^{n}\right)$. Hence, there is a model $\mathfrak{B} \vDash T$, $|B|=\aleph_{\alpha}$ and $\varphi\left(\bar{x}_{1}, \bar{x}_{2}\right)$ defines a singular equivalence relation on $B^{n}$. Therefore $\mathfrak{B} \vDash E_{0}^{n} \bar{x}_{1} \bar{x}_{2} \varphi$ and we may apply (*) again.

Case 1. For some $b \in B$, ind $\left(\varphi_{b}^{\mathfrak{B}}\right) \geq \boldsymbol{\aleph}_{0}$. Since ind $\left(\varphi_{b}^{\mathfrak{B}}\right) \leq \operatorname{ind}\left(\varphi^{\mathfrak{B}}\right)<$ $\boldsymbol{\aleph}_{\alpha}$, there is a definable singular equivalence relation on $B^{n-1}$, whence $E_{\alpha}^{n-1}$ is not eliminable in $T$. 
Case 2. ind $\left(\varphi_{b}^{\mathfrak{B}}\right)$ is finite for all $b \in B$. But then ind $\left(\pi^{\mathfrak{B}}\right) \geq \boldsymbol{\aleph}_{0}$. Also, $\operatorname{ind}\left(\pi^{\mathfrak{B}}\right) \leq\left(\operatorname{ind}\left(\varphi^{\mathscr{B}}\right)\right)^{<\omega}=\operatorname{ind}\left(\varphi^{\mathfrak{B}}\right)<\boldsymbol{\aleph}_{\alpha}$ and, therefore, $E L_{T}\left(E_{\alpha}^{1}\right)$ fails.

In either case this contradicts our original assumption and the proof is complete.

REMARK. To prove $(*)$, regularity of $\boldsymbol{\aleph}_{0}$ is needed for $\rightarrow$ and inaccessibility for $\leftarrow$. So the stronger statement in (i) also holds for all $\alpha$ such that $\aleph_{\alpha}$ is a strongly inaccessible cardinal; we do not know if it is true, e.g., for $\alpha=1$.

THEOREM 3.2. Let $T$ be stable. Suppose some quantifier $Q_{\alpha}^{m, n}$ or $E_{\alpha}^{n}$ (where $\alpha \geq 1$ and $m \geq 2$ ) is eliminable in $T$. Then $T$ is strongly regular and, hence, all $Q_{\beta}^{m, n}$ for $m, n \geq 0$ and $\beta \geq 0$ are eliminable in $T$.

Proof. By 2.1 and 2.7(i) we have

$$
E L_{T}\left(E_{\alpha}^{1}\right), \quad E L_{T}\left(E_{0}^{1}\right) .
$$

As $T$ is stable it tollows from Baldwin's and Kueker's Theorem 1.1 that $T$ does not have the finite cover property.

Now assume, for contradiction, that $\Gamma$ is not strongly regular. That is, for some $m$ and $k, T$ has an $(m, k)$-singular model. So let $Y$ be a $\lambda$-powered maximally homogeneous set of $k$-tuples for $\varphi\left(\bar{x}_{1}, \ldots, \bar{x}_{m}, \bar{y}\right)$ in $(\mathfrak{U}, \bar{a})$. Suppose $|A|=\boldsymbol{\aleph}_{\beta}, \boldsymbol{\aleph}_{0} \leq \lambda<\boldsymbol{\aleph}_{\beta}$. For notational simplicity we suppress $\bar{y}$ and $\bar{a}$.

Let $\delta\left(\bar{u}, \bar{y}_{1}, \ldots, \bar{y}_{m-1}\right)$ be the following formula, where $l(\bar{u})=l\left(\bar{y}_{i}\right)=k$ $(1 \leq i \leq m)$ :

$$
\begin{aligned}
& \delta:=\bigwedge_{1 \leq i<m} " \bar{u} \neq \bar{y}_{i} " \\
& \wedge \bigwedge_{\substack{f:\{1, \ldots, m\} \\
\rightarrow\left\{\bar{u}, \bar{y}_{1}, \ldots, \bar{y}_{m-1}\right\} \\
\bar{u} \in \operatorname{range}(f)}} \varphi\left(\bar{x}_{1} \backslash f(1), \ldots, \bar{x}_{m} \backslash f(m)\right) .
\end{aligned}
$$

Put $\Sigma:=\left\{\delta\left(\bar{u}, \bar{b}_{1}, \ldots, \bar{b}_{m-1}\right) \mid \bar{b}_{1}, \ldots, \bar{b}_{m-1} \in Y\right\}$.

As $Y$ is infinite, $\Sigma$ is consistent in $\mathfrak{A}$, i.e., for every finite $\Sigma_{0} \subset \Sigma$ : $\mathfrak{A} \vDash \exists \bar{u} \bigwedge_{\pi \in \Sigma_{0}} \pi$. Also, $\Sigma$ is omitted in $\mathfrak{A}$ since $Y$ maximally homogeneous.

Now, much like Baldwin and Kueker made use of the f.c.p.-theorem, we shall apply another result of Shelah:

TheOREM 3.3 ([4, p. 80]). Suppose $T$ does not have the f.c.p. and some $\mathfrak{M} \vDash T$ omits a $\Delta$-m-type of cardinality $\lambda$, where $\Delta$ is finite. Then there are 
$p<\omega$, a formula $\gamma\left(\bar{x}_{1}, \bar{x}_{2}, \bar{z}\right)$ with $l\left(\bar{x}_{1}\right)=l\left(\bar{x}_{2}\right)=p$ and $\bar{c} \in M$ such that

(i) $(\mathfrak{M}, \bar{c}) \vDash \mathrm{eq}(\gamma)$;

(ii) $\boldsymbol{\aleph}_{0} \leq \operatorname{ind}\left(\gamma^{(\mathfrak{R}, \bar{c})}\right) \leq \lambda$.

By (2) and the previous remarks, the hypotheses of 3.3 are satisfied with $\mathfrak{M}=\mathfrak{A}, m=k$ and $\Delta=\{\delta\}$.

Now the conclusion of 3.3 implies that, for some number $p<\omega$, $E L_{T}\left(E_{\beta}^{p}\right)$ fails. Although $p$ may be very large (as a proof of 3.3 would show), it follows from Proposition 3.1(ii) that

$$
E_{\beta}^{1} \text { is not eliminable in } T \text {. }
$$

But this leads to a contradiction: by (3) and 2.7(ii), $E L_{T}\left(E_{1}^{1}\right)$ fails. On the other hand, as $T$ is stable, (1) together with Theorem 2.9 imply $E L_{T}\left(E_{1}^{1}\right)$.

COROLlARY 3.4. Let T be stable. Then the following are equivalent:

(i) $T$ is strongly regular;

(ii) $T$ is regular;

(iii) $T$ is 1-regular for equivalence relations.

$\operatorname{Proof}($ i) $\Rightarrow$ (ii) is trivial.

(ii) $\Rightarrow$ (iii): If $\varphi(x, y)$ defines a singular equivalence relation on $A$ in some model $\mathfrak{A}$, then $\neg \varphi$ shows that $\mathfrak{A}$ is a $(2,1)$-singular model.

(iii) $\Rightarrow$ (i): By (the proofs of) 3.3 and 3.1 (i).

Acknowledgment. I would like to thank H.-D. Ebbinghaus, J. Flum and M. Ziegler for valuable comments on the subject and the presentation of this paper.

\section{REFERENCES}

[1] J. Baldwin and D. Kueker, Ramsey-quanatifiers and the finite cover property, Pacific J. Math., 90 (1980), 11-19.

[2] A. Baudisch, D. Seese, H. P. Tuschik and M. Weese, Decidability and quantifier elimination, Chapter XIX of "Higher Model Theory" (to appear).

[3] P. Rothmaler and H. P. Tuschik, $A$ two-cardinal theorem for homogeneous sets and the elimination of Malitz quantifiers. Trans. Amer. Math. Soc., 269 (1982), 273-283.

[4] S. Shelah, Classification Theory, North Holland, 1978.

[5] H. P. Tuschik, Elimination of cardinality quantifiers, ZMLG 28 (1982), 75-81.

Received October 22, 1982.

MATH. INSTITUT DER UNIVERSITÄT

ABTEILUNG FÜR LOGIK

ALBERTSTR. 23b

D-7800 Freiburg, WeSt Germany 


\section{PACIFIC JOURNAL OF MATHEMATICS EDITORS}

DONALD BABBITT (Managing Editor)
University of California
Los Angeles, CA 90024
CHARLES R. DEPrIMA
California Institute of Technology
Pasadena, CA 91125
R. FINN
Stanford University
Stanford, CA 94305

\author{
HERMANN FLASCHKa \\ University of Arizona \\ Tucson, AZ 85721 \\ RAMESH A. GANGOLLI \\ University of Washington \\ Seattle, WA 98195 \\ ROBION KIRBY \\ University of California \\ Berkeley, CA 94720 \\ C. C. Moore \\ University of California \\ Berkeley, CA 94720
}

Hugo Rossi

University of Utah

Salt Lake City, UT 84112

H. SAMELSON
Stanford University
Stanford, CA 94305

HAROLD STARK

University of California, San Diego

La Jolla, CA 92093

\section{ASSOCIATE EDITORS}
R. ARens
E. F. BECKENBACH (1906-1982)
B. H. NeUmanN
F. WOLF
K. YOSHIDA

\begin{tabular}{ll}
\multicolumn{2}{c}{ SUPPORTING INSTITUTIONS } \\
UNIVERSITY OF ARIZONA & UNIVERSITY OF OREGON \\
UNIVERSITY OF BRITISH COLUMBIA & UNIVERSITY OF SOUTHERN CALIFORNIA \\
CALIFORNIA INSTITUTE OF TECHNOLOGY & STANFORD UNIVERSITY \\
UNIVERSITY OF CALIFORNIA & UNIVERSITY OF HAWAII \\
MONTANA STATE UNIVERSITY & UNIVERSITY OF TOKYO \\
UNIVERSITY OF NEVADA, RENO & UNIVERSITY OF UTAH \\
NEW MEXICO STATE UNIVERSITY & WASHINGTON STATE UNIVERSITY \\
OREGON STATE UNIVERSITY & UNIVERSITY OF WASHINGTON
\end{tabular}

The Supporting Institutions listed above contribute to the cost of publication of this Journal, but they are not owners or publishers and have no responsibility for its content or policies.

\footnotetext{
Mathematical papers intended for publication in the Pacıfic Journal of Mathematıcs should be in typed form or offset-reproduced (not dittoed), double spaced with large margins. Please do not use built up fractions in the text of the manuscript. However, you may use them in the displayed equations. Underline Greek letters in red, German in green, and script in blue. The first paragraph must be capable of being used separately as a synopsis of the entire paper. In particular it should contain no bibliographic references. Please propose a heading for the odd numbered pages of less than 35 characters. Manuscripts, in triplicate, may be sent to any one of the editors. Please classify according to the scheme of Math. Rev ews, Index to Vol. 39. Supply name and address of author to whom proofs should be sent. All other communications should be addressed to the managing editor, or Elaine Barth, University of California, Los Angeles, California 90024.

There are page-charges associated with articles appearing in the Pacific Journal of Mathematics. These charges are expected to be paid by the author's University, Government Agency or Company. If the author or authors do not have access to such Institutional support these charges are waived. Single authors will receive 50 free reprints; joint authors will receive a total of 100 free reprints. Additional copies may be obtained at cost in multiples of 50 .
}

The Pacific Journal of Mathematics is issued monthly as of January 1966. Regular subscription rate: $\$ 190.00$ a year (5 Vols., 10 issues). Special rate: $\$ 66.00$ a year to individual members of supporting institutions.

Subscriptions, orders for numbers issued in the last three calendar years, and changes of address should be sent to Pacific Journal of Mathematics, P.O. Box 969, Carmel Valley, CA 93924, U.S.A. Old back numbers obtainable from Kraus Periodicals Co., Route 100, Millwood, NY 10546.

The Pacific Journal of Mathematics at P.O. Box 969, Carmel Valley, CA 93924 (ISSN 0030-8730) publishes 5 volumes per year. Application to mail at Second-class postage rates is pending at Carmel Valley, California, and additional mailing offices. Postmaster: Send address changes to Pacific Journal of Mathematics, P.O. Box 969, Carmel Valley, CA 93924.

PUBLISHED BY PACIFIC JOURNAL OF MATHEMATICS, A NON-PROFIT CORPORATION Copyright $\odot 1985$ by Pacific Journal of Mathematics 


\section{Pacific Journal of Mathematics}

\section{Vol. 117, No. 2 \\ February, 1985}

Robert Walter Bagley, Ta-Sun Wu and J. S. Yang, On a class of topological groups more general than SIN groups ............... 209

Bruce Alan Barnes, Algebraic elements of a Banach algebra modulo an ideal ...............................................219

Howard D. Fegan and Peter Gilkey, Invariants of the heat equation .......233

Erica Flapan, Necessary and sufficient conditions for certain homology

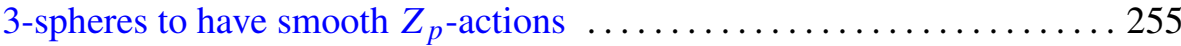

Philip R. Heath, Product formulae for Nielsen numbers of fibre maps . . . . 267

Derbiau Frank Hsu and A. Donald Keedwell, Generalized complete mappings, neofields, sequenceable groups and block designs. II ...... 291

Taqdir Husain, Orthogonal primitive idempotents and Banach algebras

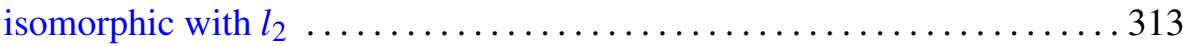

Gary M. Lieberman, Regularized distance and its applications . . . . . . . 329

William W. Menasco, Determining incompressibility of surfaces in alternating knot and link complements ............................ 353

Benjamin Muckenhoupt, Weighted reverse weak type inequalities for the Hardy-Littlewood maximal function $\ldots \ldots \ldots \ldots \ldots \ldots \ldots \ldots \ldots \ldots \ldots \ldots$

John Dacey O'Neill, Direct summands of direct products of slender

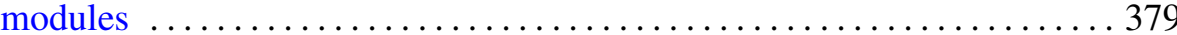

Andreas Rapp, Elimination of Malitz quantifiers in stable theories 387

Francisco José Ruiz, A unified approach to Carleson measures and $A_{p}$ weights

Hanamatagouda Pandappa Sankappanavar, Heyting algebras with dual

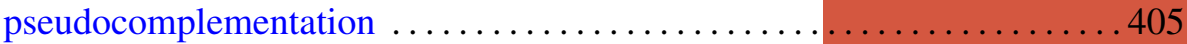

Annette Sinclair, Strong Carleman and strong uniform approximation . . . . 417

Masaaki Suzuki, The generalized Schwarz lemma for the Bergman metric

Brian Thorpe and Ludwig Tomm, Universal approximation by regular

weighted means 